\title{
A DIETA E O CONSUMO DIÁRIO DE ALIMENTO DE Cichla monoculus NA AMAZÔNIA CENTRAL.
}

\author{
Hermógenes RABELO, Carlos A.R.M. ARAÚJO-LIMA ${ }^{1}$
}

RESUMO - Avaliar a dieta, o ritmo alimentar e a taxa de consumo diária de alimento dos peixes pode permitir estimar a relação entre a alimentação e o crescimento, a pressão de predação sobre espécies de presas, a limitação alimentar durante estações do ano e a competição intra e interespecífica. Estas informações são desconhecidas para C.monoculus na Amazônia Central e aqui são apresentados dados sobre a ecologia trófica desta espécie durante quatro estações hidrológicas. A área de estudo incluía três lagos de várzea na Amazônia Central, durante os períodos de agosto de 1997 a julho de 1998. Os estudos da dieta e do ritmo alimentar foram feitos através das análises dos conteúdos estomacais. A taxa de evacuação gástrica foi estimada experimentalmente. $\mathrm{O}$ consumo diário de alimento foi calculado a partir dos modelos de Elliot \& Persson e de Eggers. A intensidade de alimentação da espécie foi baixa durante a seca. A dieta do C. monoculus foi basicamente piscívora e composta de nove famílias de peixes e uma de camarão, apresentando variações no decorrer das estações hidrológicas e com o tamanho do peixe. A taxa de evacuação gástrica foi $16,9 \% \mathrm{~h}^{-1}$. O consumo diário de alimento, que não foi diferente nas quatro estações hidrológicas, teve média igual a $2,23 \%$ do peso corporal. Este valor é baixo comparado a outros estudos estimados para peixes tropicais. Indicando que esta espécie come relativamente pouco.

Palavras-chave: Cichla, Amazônia, peixes, alimentação, consumo diário

Feeding and Daily Ration of Cichla monoculus in Central Amazon.

ABSTRACT - Information on food, feeding rhythm and daily ration of fish are useful to evaluate the relationship between growth and feeding, predation pressure, competition, and seasonal food limitation. This type of information was lacking to Cichla monoculus in its natural habitats in the Central Amazon floodplain and here it is presented data on the feeding ecology of this species during one hydrological cycle. The study included three floodplain lakes sampled between August 1997 and July 1998. The diet and feeding rhythm were evaluated with gut content analysis and the gastric evacuation rate was measured experimentally. The daily ration was estimated with Elliot and Persson's model and Egger's model. Feeding intensity was lower during the dry season. The diet consisted basically of fish, comprising 9 families of fish but one family of shrimp occurred in $20 \%$ of the samples. The gastric evacuation rate was $16.9 \% \mathrm{~h}^{-1}$. The daily ration was not different between seasons and averaged $2.23 \%$ bw. This is a relatively low value compared to other piscivores.

Key-words: Cichla, food, gastric evacuation, daily ration, Amazon, fish

\section{Introdução}

A análise das dietas dos peixes é importante para estudos de predação, competição e cadeia alimentar aquática. A dieta pode ser estudada através da observação direta do comportamento alimentar e/ou pela identificação do conteúdo estomacal, refletindo a disponibilidade de alimento num dado ambiente ou período do ano (Andrian \& Barbieri, 1996; Moreno-Amich, 1996) e mudanças na alimentação ao longo da vida em conseqüência de modificações ontogenéticas (Wootton, 1990; Oliveros \& Rossi, 1991; Shimamoto \& Watanabe, 1994).

O tucunaré comum, Cichla monoculus Spix (1831) é um importante

${ }^{1} \mathrm{INPA}$, Av. André Araujo 1756, Manaus, Am, 69083-000 
predador da Amazônia Central. Nos locais onde foi estudada, incluindo aqueles onde ela foi introduzida, esta espécie sempre apresentou um hábito predominante piscívoro. Cala et al. (1996) encontraram nos estômagos do C. monoculus, no alto rio Solimões, em diferentes fases de crescimento, 13 categorias definidas de itens alimentares, mas os indivíduos maiores ingeriram principalmente peixe. Arcifa \& Meschiatti (1993) registraram variação ontogenética nos itens alimentares do tucunaré Cichla ocellaris no reservatório de Monte Alegre. Indivíduos menores que $8 \mathrm{~cm}$ alimentavam-se basicamente de insetos independente de sazonalidade, e peixe era o principal alimento dos adultos.

Em geral o tamanho da presa tem uma relação direta com o tamanho do predador (Gerking, 1994). A maioria dos peixes predadores na várzea do rio Solimões consomem presas que chegam a medir até $40 \%$ do seu comprimento (Araújo-Lima et al., 1995), mas espécies de Cichla, em outros locais, podem exceder esse limite. Por exemplo, C. ocellaris, no rio Machado, ingere presas de até $57 \%$ do seu comprimento padrão (Goulding, 1980), enquanto que $C$. temensis, no rio Cinaruco, Venezuela, captura presas de $45 \%$ do seu comprimento (Winemiller et al., 1997).

A dieta do C. monoculus em lagos de várzea da Amazônia Central nunca foi sistematicamente abordada. Esta espécie também deve ser piscívora nesta região, mas é possível que sua dieta apresente algumas variações, incluindo aquelas de ordem sazonal. Também é possível que o tamanho das presas consumidas por $C$. monoculus exceda a amplitude encontrada para os piscívoros do rio Solimões, como já foi registrado em outras comunidades de peixes.

O ritmo alimentar refere-se ao comportamento alimentar durante um ciclo, o qual é característico de cada indivíduo, espécie e/ou população. Ele é denominado de acordo com a escala temporal que está sendo analisada. Um primeiro caso corresponde ao ciclo de 24 horas ou ritmo diário em que o alimento mais importante é consumido; outro seria o ciclo anual, que pode variar de acordo com as estações do ano, ou com a flutuação do nível da água de um rio.

O ritmo diário é a forma cíclica mais comum entre as espécie que vivem influenciadas pelos efeitos do dia e da noite. Jenkin Jr (1969) identificou que as trutas e salmões apresentam, em sua maioria, ritmo alimentar diurno. Bordeur \& Pearcy (1987) estudando juvenis de Oncorhynchus kisutch sugeriram que o ritmo alimentar diário é usualmente caracterizado por dois picos, um pela manhã, $\log o$ ao alvorecer do dia e outro pela tarde, ao por do sol. Eles demonstraram que o nível máximo de peso do alimento em relação ao peso corporal ocorreu no início da noite. Situação idêntica foi descrita em duas espécies de pescadas, Plagioscion squamosissimus e $P$. montei, na Amazônia Central (Worthmann \& Oliveira 1987). Em Prenesus pinguis, 
o ritmo dos jovens é diurno e dos adultos noturno (Hobson \& Chess, 1973).

Em um ciclo anual, os eventos fisiológicos da alimentação seguem o ritmo imposto pelas características sazonais do ambiente, as quais mudam diariamente ao longo do ciclo, fazendo com que tais eventos acompanhem essas mudanças. Em juvenis de Oncorhynchus nerka, por exemplo, a maior parte da população não se alimenta durante o inverno e apenas poucos indivíduos ingerem algum alimento (Doble \& Eggers, 1978). O mesmo pode acontecer em ambientes sazonais tropicais. Peixes carnívoros do pantanal mato-grossense, por exemplo, apresentam variação no índice alimentar ao longo de um ciclo hidrológico (Resende et al., 1996).

A reprodução também modifica o ritmo alimentar, principalmente em peixes que apresentam cuidado parental utilizando a cavidade orobranquial, como machos e fềmeas de Cichla ocellaris (Zaret, 1980), ou em peixes que preparam e tomam conta dos ninhos, como os machos de Gasterosteus aculeatus (Noakes, 1986). O ritmo alimentar e o índice de repleção do C. monoculus podem ser dependentes de variações sazonais do ambiente, como as estações hidrológicas, que influenciam a oferta de alimento e condicionam as épocas de reprodução dos peixes.

O conhecimento da taxa de consumo diário de alimento dos peixes pode ser utilizado para estimar a relação entre a alimentação e o crescimento, a pressão de predação sobre espécies de presas, a limitação alimentar durante estações do ano e a competição intra e inter-específica (Nikolsky, 1978; Ney, 1990). Esta taxa é estimada pela combinação de dados de conteúdo estomacal em ambientes naturais e a taxa de evacuação gástrica do alimento.

A evacuação gástrica inicia a partir da ingestão do alimento. Geralmente modelos exponenciais, lineares e raiz quadrada são usados para descrever a relação entre o resto de alimento no estômago e tempo passado após a ingestão (Elliot, 1972; Elliot \& Persson, 1978; Persson, 1979; Jobling, 1981; Jobling, 1986; Mullen, 1986; Olson \& Mullen, 1986; Persson, 1986). Atualmente a taxa de evacuação gástrica medida experimentalmente é um dos principais parâmetros utilizados para estimar a taxa de consumo de alimento de peixes em ambientes naturais.

A combinação da composição, forma e tamanho da presa, com a fisiologia do predador e a temperatura, pode influenciar a taxa de evacuação gástrica (Persson, 1979; Jobling, 1987; Salvanes et al., 1995). A temperatura é o principal fator abiótico que age sobre a taxa de metabolismo dos peixes, e consequentemente no consumo de alimento e no processo digestivo (Smith, 1989). Singh-Renton \& Bromley (1996) estudando pequenos gadóides, concluíram que a temperatura influencia a taxa de evacuação gástrica e o nível da saciação da alimentação e também que altas temperaturas estimulam os peixes a consumirem refeições maiores. 
Nestas espécies, por exemplo, a refeição é de $5,8 \%$ do peso corporal a $6{ }^{\circ} \mathrm{C}$ e $8 \%$ do peso corporal a $12,7^{\circ} \mathrm{C}$.

$\mathrm{O}$ consumo diário de alimento de peixes piscívoros tropicais varia entre 4 a $36 \%$ e de peixes da zona temperada varia entre 0,5 a $17 \%$ em relação ao peso corporal fresco (Pandian \& Vivenkanadan, 1985). Por exemplo, Serrassalmis nattereri, peixe neotropical, apresentou um consumo de $12,1 \%$ do peso corporal numa temperatura de $27{ }^{\circ} \mathrm{C}$ (Pauly, 1994). Hall (1987) estudando consumo diário de alimento em Melanogrammus aeglefinus e Limanda limanda, em temperatura controlada de $12{ }^{\circ} \mathrm{C}$, estimou que a taxa de consumo em relação ao peso corporal foram de $6,0 \mathrm{e}$ $4,1 \%$, respectivamente, para cada espécie. O salmão Oncorhynchus ksuitch consome 2,4 e $3,7 \%$ do peso corporal na temperatura de 11,4 e $13,7{ }^{\circ} \mathrm{C}$, respectivamente (Brodeur \& Pearcy, 1987).

O consumo de alimento de peixes neotropicais ainda é relativamente pouco conhecido. Provavelmente, C. monoculus apresenta uma taxa de consumo diário de alimento próximo aos níveis de consumo médio de $16,7 \%$ ( $\mathrm{SE}=$ 2,042) citado para peixes tropicais (Pandian \& Vivekanandan, 1985).

O objetivo do presente trabalho foi conhecer a variação na dieta durante um ciclo hidrológico e determinar o consumo diário de alimento de $C$. monoculus na Amazônia Central. Estas informações ainda não estão disponíveis para esta espécie e permitirá uma melhor avaliação do seu papel como predador nos ecossistemas em que habita

\section{Materiais e métodos}

As amostragens foram realizadas mensalmente, de agosto de 1997 a julho de 1998, de três lagos de várzea na Amazônia Central: Janauacá ( $3^{\circ} 20^{\circ}$ $\mathrm{S}$ e $60^{\circ} 20^{\prime} \mathrm{W}$ ), Camboa (3' $12^{\prime} \mathrm{S}$ e $\left.59^{\circ} 35^{\prime} \mathrm{W}\right)$ e dos Reis ( $3^{\circ} 08^{\prime} \mathrm{S}$ e $59^{\circ}$ $29^{\prime}$ W). Os tucunarés, C. monoculus, foram amostrados em períodos de 24 horas em áreas recomendadas por pescadores profissionais. As capturas diurnas foram feitas com redes de espera, de malhas de $40,50,60,70$, $80,90,100$ e 110 milímetros entre nós opostos, 25 metros de comprimento e 3 metros de altura, dispostas às margens do lago, sendo examinadas em intervalos de 3 horas. Nas áreas rasas, livres de troncos e galhos de árvores submersos, as capturas foram realizadas com rede de arrasto de 15 metros de comprimento. A noite as capturas foram feitas com rede de espera e arpão, pois neste período os peixes apresentavam pouca mobilidade, dificultando a captura, e por isto aumentamos o esforço.

Imediatamente após a captura, os exemplares foram dissecados, para retirada dos estômagos e em seguida pesados $(0,1 \mathrm{~g})$ e medidos (comprimento padrão; $0,1 \mathrm{~cm}$ ). Os estômagos foram etiquetados (local, data e hora de captura, peso e comprimento do peixe), conservados em frascos com formol a $10 \%$, e transportados para o Laboratório de Ecologia de Peixes II do INPA, onde foram passados para álcool a $70 \%$ após 2 dias. 
No laboratório os estômagos foram abertos para identificar a composição do conteúdo estomacal. Os itens alimentares foram medidos com paquímetro $(0,1 \mathrm{~cm})$ e pesados $(0,0 \mathrm{lg})$. $\mathrm{O}$ peso do conteúdo estomacal foi calculado como porcentagem do peso corporal do tucunaré. Foram assumidos como estômagos vazios somente os que continham menos de $10 \%$ do peso máximo encontrado nos conteúdos estomacais.

Os dados foram agrupados em quatro estações hidrológicas para facilitar as análises, sendo considerados como enchente os meses de fevereiro a abril, cheia os meses de maio a julho, vazante os meses de agosto a outubro e seca os meses de novembro a janeiro, mesmo padrão usado em outros estudos na Amazônia Central (Santos, 1980; Goulding et al., 1988; Odinetz-Collart \& Moreira, 1993). As variações das proporções de estômagos vazios $(<10 \%)$ e das freqüências de ocorrência das presas com as estações hidrológicas foram testados com testes $\mathrm{G}$. A variação na composição da dieta e o comprimento padrão do tucunaré foram analisados com a correlação não-paramétrica $\left(\mathrm{r}_{\mathrm{s}}\right)$ de Spearman. O comprimento das presas em relação ao comprimento padrão $(\mathrm{cm})$ do tucunaré foi ajustado por regressão linear com o método dos mínimos quadrados.

A partir do comprimento padrão de cada presa identificada no conteúdo estomacal, foi estimado o peso da presa no momento em que foi ingerida pelo tucunaré através de regressões peso/ comprimento (Swenson \& Smith, 1973).
Estas regressões foram calculadas com amostras de presas da mesma espécie ou gênero, capturadas no lago Janauacá. A hora de ingestão de cada presa foi retrocalculada do peso digerido através da taxa de evacuação gástrica. Apenas peixes cujas presas pudessem ser identificadas, foram usados nesta análise. $\mathrm{O}$ peso médio relativo do conteúdo estomacal foi calculado, para cada estação hidrológica, em intervalos de 3 horas em ciclos de 24 horas.

A taxa de evacuação gástrica foi medida experimentalmente. Os experimentos foram realizados na Estação de Piscicultura da Unidade Hidrelétrica da Balbina, Município de Presidente Figueiredo, Amazonas, em oito tanques de concreto de $3 \mathrm{~m}^{3}$. Os tanques foram protegidos contra a incidência direta de raios solares. $\mathrm{O}$ sistema de circulação de água foi contínuo, usando água do reservatório. A temperatura da água dos tanques, que foi monitorada a cada hora durante os experimentos, variou de 27,6 a $30,2{ }^{\circ} \mathrm{C}$ (média $=29,4{ }^{\circ} \mathrm{C}$ ).

Os peixes foram capturados no reservatório da UHE de Balbina, próximo à estação de piscicultura, usando anzóis sem farpa, para evitar ferimentos excessivos. Estes passaram por um período de aclimatação de aproximadamente uma semana, alimentados ad libitum, com Tilapia rendalli, para que recuperassem do estresse do transporte e captura e se alimentassem regularmente. Os experimentos foram realizados com 24 peixes cujos pesos e comprimentos variaram de 202,0 a 456,0 g (média $=$ $308,9 \mathrm{~g}$ ) e 22,3 a $30,4 \mathrm{~cm}$ (média $=$ 
$26,9 \mathrm{~cm})$, respectivamente. A preparação para os experimentos consistiu de um jejum pré-prandial de $72 \mathrm{~h}$, para que houvesse total evacuação gástrica de eventuais resíduos de alimento.

A cada tucunaré foi oferecido um exemplar de $T$. rendalli, com peso aproximado de $3 \%$ do predador $(3,93$ a $12,22 \mathrm{~g}$ ), e com nadadeira anal cortada, para dificultar o natação. A tilápia era amarrada por uma linha na superfície do tanque. $\mathrm{O}$ horário exato de ingestão era indicado pela tensão da linha, que era imediatamente cortada para liberar a presa. Os tucunarés foram abatidos em intervalos de tempo medidos a partir do momento da ingestão, com duração de 3 a 17 horas. Ao final de cada intervalo o conteúdo gástrico de cada tucunaré foi removido e pesado, após a retirada do excesso de umidade com papel filtro.

A taxa de evacuação gástrica horária foi estimada pelo método dos mínimos quadrados usando regressão linear após transformação logarítmica de $\mathbf{A}_{\mathbf{h}}$ (Elliot 1972; Persson 1986). O modelo exponencial resultante foi:

$$
A_{h}=A_{0}^{*} e^{-(\mathrm{R} * h)},
$$

onde: $\mathbf{A}_{\mathbf{h}}$ foi a porcentagem de alimento restante no estômago do tucunaré no momento do abate; $h$ foi o tempo em horas medido a partir da ingestão da presa até o abate; $\mathbf{A}_{0}$ foi o peso inicial da presa; e $\mathbf{R}$ foi a taxa instantânea de evacuação gástrica. A escolha deste modelo foi baseada nas premissas teóricas de que o peixe não se alimenta continuamente durante um ciclo e que a quantidade de alimento no estômago diminui de acordo com o volume do alimento (Persson, 1986). Os efeitos do tamanho da presa, da temperatura da água e do tamanho do tucunaré sobre a evacuação gástrica foram analisados pela inspeção gráfica dos resíduos.

$\mathrm{O}$ consumo diário de alimento foi medido a partir de dois modelos exponenciais nas quatro estações hidrológicas.

1) modelo de Elliot \& Persson (1978);

$$
\mathrm{C}_{\mathrm{t}}=\sum_{\mathbf{i}=\mathbf{1}}^{\mathrm{m}} \mathrm{C}_{\mathrm{ti}}
$$

onde,

$C_{t i}=\left[\left(A_{m}-A_{m-1} e^{-R t}\right) R t\right] /\left(1-e^{-R t}\right)$

sendo $\mathbf{A}_{\mathrm{m}}$ peso relativo do alimento no final do intervalo $\mathbf{m}, \mathbf{A}_{\mathbf{m}-1}$ peso relativo do alimento no início do intervalo $\mathbf{m}, \mathbf{R}$ taxa instantânea de evacuação gástrica, $\mathbf{t}$ igual a 3 horas. $\mathbf{A}_{\mathrm{m}}$ foi estimado com coletas regulares durante os meses de agosto 1997 a julho de 1998, para cada intervalo de 3 horas.

2) modelo de Eggers (1979):

$$
C_{t}=24 . R \cdot S
$$

onde $\mathbf{R}$ é a taxa instantânea de evacuação gástrica e $\mathbf{S}$ o peso médio relativo do conteúdo estomacal durante um período de 24 horas.

Os consumos diários nas quatro estações hidrológicas foram comparados através da ANOVA não paramétrica de Friedman.

\section{Resultados}

Foram amostrados 171 exemplares de C. monoculus, sendo a maior captura, 81 peixes $(47.4 \%$ do 
total), nos meses da vazante (agosto, setembro e outubro) e a menor, 13 peixes $(7,6 \%$ do total), nos meses da enchente (fevereiro, março e abril) (Tab. I). O comprimento padrão e o peso dos tucunarés capturados variaram de 12,3 a $41,2 \mathrm{~cm}$ (média $=26,6 \mathrm{~cm}$ ) e de 105,6 a 1350,2 $\mathrm{g}$ (média $435,2 \mathrm{~g}$ ), respectivamente.

Dentre os estômagos analisados, $58,3 \%$ estavam vazios. A proporção de estômagos vazios esteve relacionada ao ciclo hidrológico (teste $\mathrm{G} ; \mathrm{P}<0,001$; Fig. I). A dieta consistiu somente de peixes e camarões, cujas freqüências de ocorrência médias foram $78,9 \%$ e $21,1 \%$, respectivamente. Em dois estômagos foram encontrados restos de macrófitas aquáticas, que provavelmente haviam sidas ingeridas de forma acidental. As frequiências de ocorrência de camarão nos estômagos do tucunaré estavam inversamente correlacionadas com o tamanho do peixe $(\mathrm{r}=-0,93$; $\mathrm{P}<0,05$ ), e variaram com as fases do ciclo hidrológico (teste $\mathrm{G}, \mathrm{P}<0,0001$. Fig. 2). Nenhum peixe maior que $35 \mathrm{~cm}$ predou camarão.

Tabela 1: Local, número de individuos e amplitude de comprimento $(\mathrm{cm})$ de $C$. monoculus amostrados durante 4 perídos hidrológicos em 1997 e 1998.

\begin{tabular}{lccc}
\hline Ciclo & local & $n^{\circ}$ de individuos & amplitude \\
\hline vazante & Camboa/Rel & 81 & 18,6 a 40,6 \\
seca & Camboa/Rel & 36 & 17,0 a 41,2 \\
enchente & Camboa/Rei/Janauacá & 16 & 22,4 a 32,5 \\
cheia & Camboa/Janauacá & 38 & 12,3 a 36,6 \\
lotal & & 171 & \\
\hline
\end{tabular}

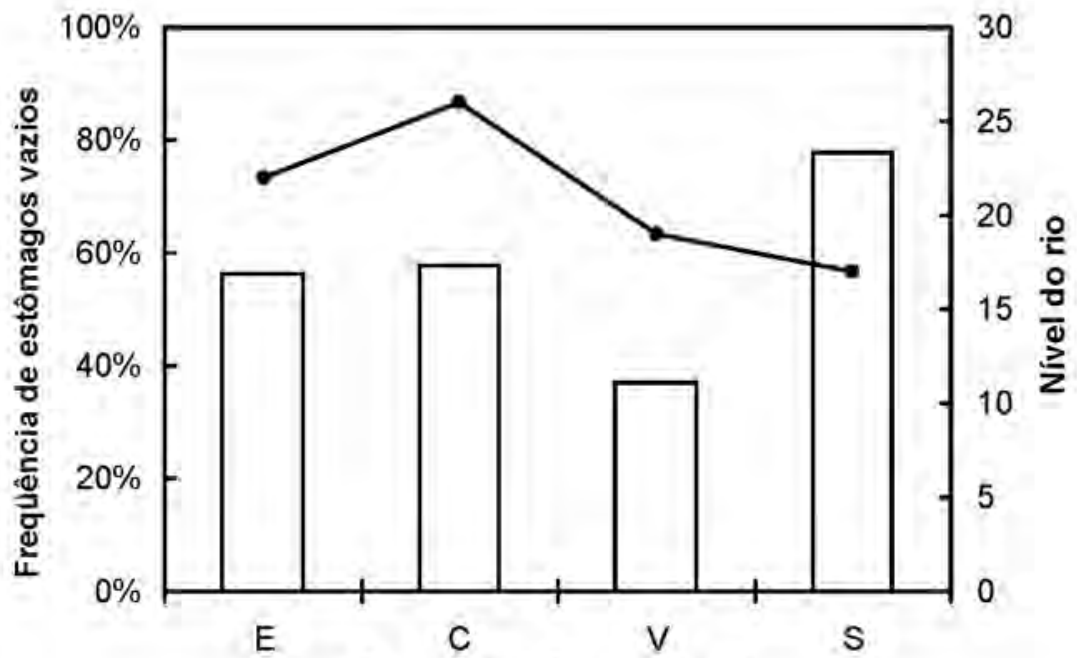

Fases do ciclo hidrológico

Figura 1. Frequência de estòmagos vazios por fase do ciclo hidrológico (teste $\mathrm{G}$; $\mathrm{p}<0,001$; gl=3: $\mathrm{n}=89$ ). 
Apenas 32,7\% das presas foram possiveis de serem identificadas. Estas pertenceram a nove famílias de peixes (Serrassalmidae, Curimatidae, Pimelodidae, Erythrinidae, Hemiodidae, Callichthyidae, Loricariidae, Anastomidae e Prochilodontidae) e uma família de decápodo (Palaemonidae). Os peixe somam $86,6 \%$ do peso da dieta. As principais espécies de presas identificadas nos conteúdos foram Hoplosternum litoralle, Serrassalmus sp, Potamorhina sp, Hoplias malabaricus, Prochilodus nigricans, Psectrogaster sp, Schizondon fasciatum e Semaprochilodus insignis. A freqüência de ocorrência das presas, entretanto, dependia do ciclo hidrológico (teste $\mathrm{G} ; \mathrm{P}<0,001$ ). Houve uma tendência de predação de Curimatidae, Serras-salmidae e Hemiodontidae na enchente/cheia. A dieta na vazante/seca foi mais variada, predominando Serrassalmidae, Erythrinidae, Pimelodidae e Callichthyidae (Fig. 3).

$\mathrm{O}$ comprimento das presas variou de 2,63 a $9,87 \mathrm{~cm}$. O tamanho das presas aumentou significativamente com o comprimento do tucunaré sendo o comprimento da presa $=-1,81+0,29 *$ comp. do tucunaré $(\mathrm{n}=38 ; \mathrm{r}=0,57 \mathrm{e}$ $\mathrm{P}<0,001)$. Nos peixes onde as presas estavam poucas digeridas, foi possível notar que o peso relativo do conteúdo estomacal foi independente do número de presas ingeridas. $\mathrm{O}$ número mediano de presa nos estômagos foi igual a 1 . Os horários de maior atividade de captura de alimento, retrocalculados a partir das relações peso/comprimento (Tab. 2), foram de 9:00 às 11:00 e 18:00 às 20:00 (Fig. 4). Tendências semelhantes foram observadas com os pesos dos conteúdos estomacais não corrigidos.

Os estômagos com alimento muito digerido foram os mais freqüentes. $\mathrm{O}$ peso médio do conteúdo estomacal durante a enchente/cheia foi $1,038+1,169 \%$ e durante a vazante/ seca foi $0,889+0 \quad 97 \%$ sendo distribuído assimetricamente e

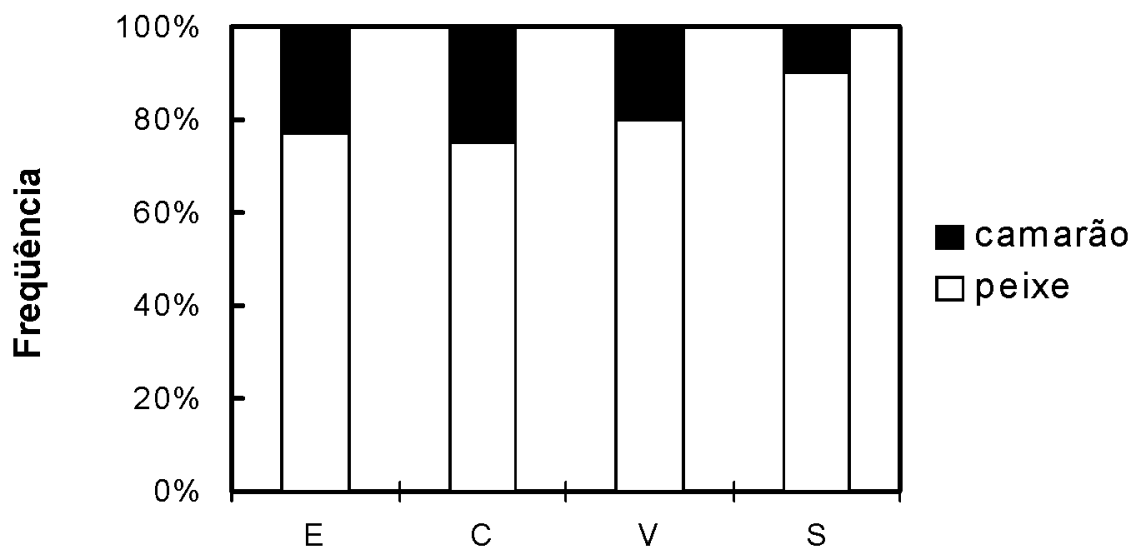

Fases do ciclo hidrológico

Figura 2. Frequência de ocorrência de camarão e peixes encontrados nos estômagos por fase do ciclo hidrológico (teste $\mathrm{G} ; \mathrm{p}<0,001 ; \mathrm{gl}=3 ; \mathrm{n}=38$ ). 


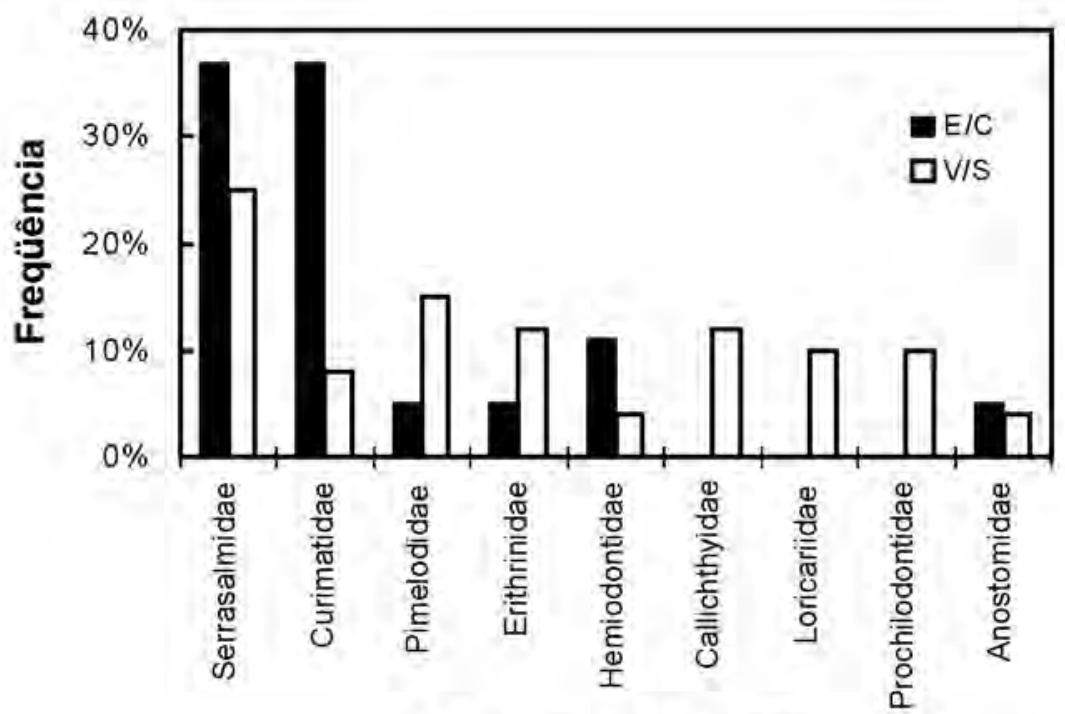

Familia das presas

Figura 3. Frequéncia de ocorrência das familias das presas (peixes) encontradas nos estômagos ern duas fases agrupadas do ciclo hidrológico (teste $\mathrm{G} ; \mathrm{p}<0,001 ; \mathrm{gl}=8 ; \mathrm{n}=9$ ).

Tabela 2: Relaçấo peso/comprimento e media e desvio padrâo (SD) de seis espécies de presas do $C$. monoculus.

\begin{tabular}{|c|c|c|}
\hline Espécies & Relação peso/comprimento & Mèdia $\pm S D(\mathrm{~cm})$ \\
\hline \multirow[t]{3}{*}{ Semaprochilodus insignis } & peso $=0,029^{\circ} \operatorname{comp}^{2.888}$ & $6,34 \pm 1,05$ \\
\hline & $r=0,97$ & \\
\hline & $n=15$ & \\
\hline \multirow[t]{3}{*}{ Potamorhina $5 p$} & peso $=0,03^{\circ} \operatorname{comp}, 7,89 x$ & $6.09 \pm 2.08$ \\
\hline & $r=0,98$ & \\
\hline & $n=9$ & \\
\hline \multirow[t]{3}{*}{ Serrassalmus sp } & peso $=0,04^{*}$ comp. ${ }^{1,949}$ & $4,67 \pm 0,71$ \\
\hline & $\mathrm{T}=0,98$ & \\
\hline & $n=14$ & \\
\hline \multirow[t]{3}{*}{ Hoplias malabaricus } & peso $=0,013^{\circ}$ comp ${ }^{s / 42^{\prime}}$ & $5,46 \pm 3,48$ \\
\hline & $r=0.99$ & \\
\hline & $n=14$ & \\
\hline \multirow[t]{3}{*}{ Schizodon fascialum } & peso $=0,015^{*}$ comp $p^{2.3 i 1}$ & $5,23 \pm 1,2$ \\
\hline & $r=0,93$ & \\
\hline & $n=13$ & \\
\hline \multirow[t]{3}{*}{ Hoplosternum thoracatum } & peso $=0,016^{*} \operatorname{comp} .^{3,292}$ & $6,39 \pm 0,77$ \\
\hline & $y=0,91$ & \\
\hline & $n=8$ & \\
\hline
\end{tabular}


significativamente diferentes nos dois períodos (teste $\mathrm{G} ; \mathrm{P}<0,001$ ).

A taxa de evacuação gástrica calculada pelo modelo exponencial foi de 0,169 ou $16,9 \% \mathrm{~h}^{-1} \quad$ (erro padrão $=0,013 ; \mathrm{gl}=22, \mathrm{P}<0,0001)$. $\mathrm{O}$ ajuste da regressão foi bom $\left(\mathrm{r}^{2}=0,90\right.$; Fig. 5). $\mathrm{O}$ valor encontrado do $\ln (\mathrm{A} 0)$ foi 5,2 (erro padrão $= \pm 0,14$ ). Os resíduos da regressão não apresentaram nenhuma tendência com o peso estimado do alimento, com a temperatura média da água durante os experimentos, e com o peso do tucunaré e das presas. Assim foi assumido que estes fatores não tiveram influência na taxa de evacuação gástrica. $O$ consumo diário de alimento do $C$. monoculus calculado com o modelo de Elliot \& Persson (1978) foi de $1,82,2,52,2,45$ e 2,04; e com o modelo de Eggers (1979) foi $1,89,2,54,2,53$ e 2,05 e não apresentaram diferenças significativas
(ANOVA não paramétrica de Friedman; $\mathrm{P}=0,47 ; \mathrm{gl}=3$ ) para as estações hidrológicas enchente, cheia, vazante e seca, respectivamente.

\section{Discussão}

O número de $C$. monoculus capturados variou sazonalmente sendo maior na vazante e menor na enchente, mas foi suficiente para analisar a dieta, nas quatro estações. A maioria dos peixes estudados foi maior que $20 \mathrm{~cm}$, ou seja, tinham atingido a maturidade sexual, segundo Correa (1998). Esta espécie tem um longo período de reprodução, que se estende da seca à enchente.

Goulding (1980) e Araujo-Lima et al. (1995) sugeriram que a oferta de alimento, a uma comunidade de peixes, é dependente de variações ambientais, principalmente em relação ao nível da água do rio. Durante a enchente e cheia o nível da água permite

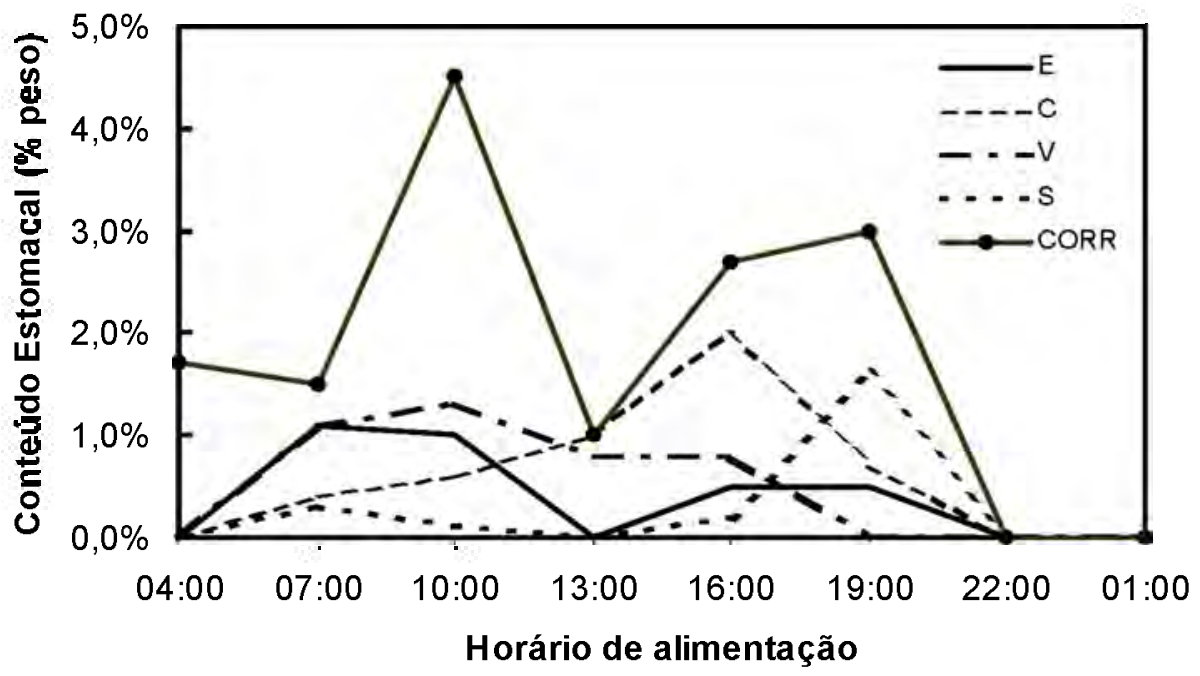

Figura 4. Variação horária do peso relativo observado $(n=82)$ e corrigido $(n=27)$ do conteúdo estomacal durante $24 \mathrm{~h}$ em quatro fases do ciclo hidrológico. 


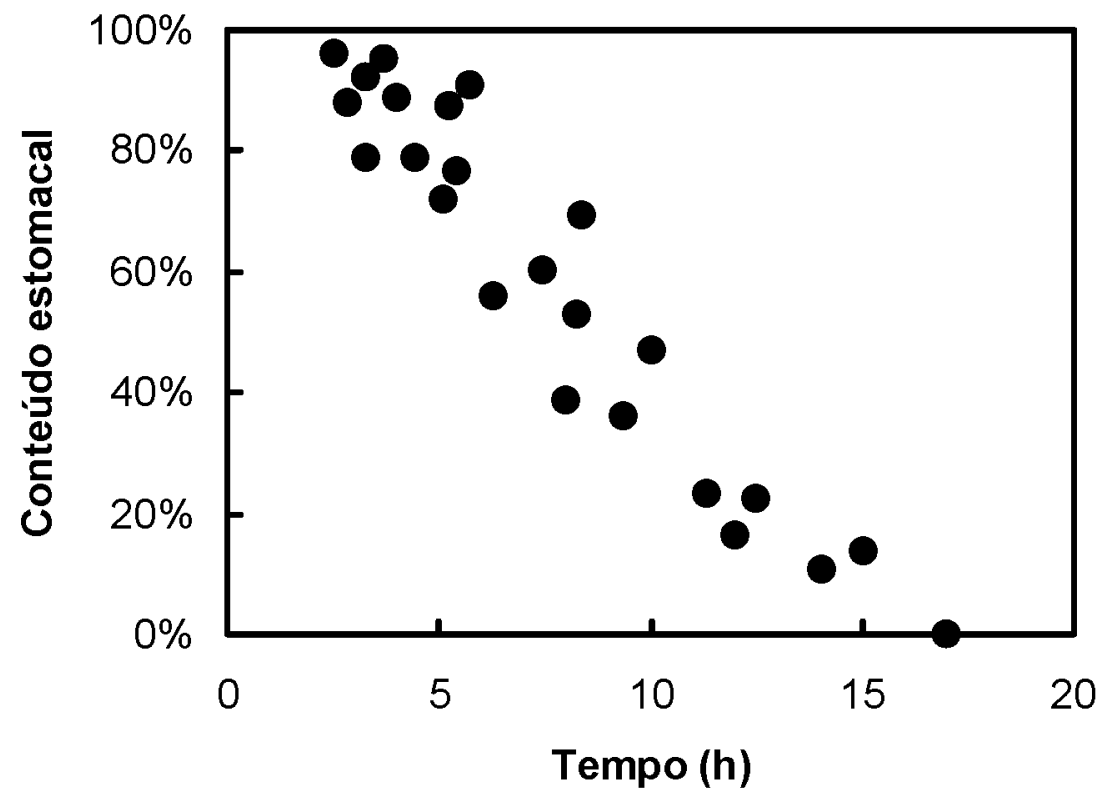

Figura 5. Redução do conteúdo estomacal relatıvo com o tempo pós-prandıal em condıções experimentais $\left(\mathrm{r}^{2}=0,90 ; \mathrm{p}=0,0001\right)$

o acesso dos peixes à floresta alagada, criando um ambiente que favorece os predadores que tocaiam suas presas, como o tucunaré. Durante a seca o espaço é reduzido e todas as espécies se concentram no lago, o que favorece aos piscívoros (Lowe-McConnell,1987; De Merona \& Bittencourt, 1993). Um outro fator que poderia influenciar a alimentação dos peixes é a reprodução. Os tucunarés comem menos após a desova devido ao seu hábito de proteger a prole (Zaret, 1980).

A intensidade de alimentação e/ ou oferta de alimento pode ser refletida pela quantidade de estômagos vazios numa população. No presente trabalho a freqüência de estômagos vazios do $C$. monoculus foi em média $53,8 \%$ e esteve relacionada ao nível do rio, sendo esta mais alta durante a seca. Porém, a quantidade de estômagos vazios contrasta com a hipótese de aumento de densidade de presas com a seca, que elevaria a oferta de alimento. Possivelmente o jejum durante a reprodução e a ausência da floresta inundável exercem maior influência sobre a intensidade de alimentação da espécie.

Os resultados de outros estudos não mostram um padrão para a espécie. Cala et al. (1996) analisando conteúdo estomacal de $C$. monoculus, em lagos próximo a fronteira do Brasil com a Colômbia, encontraram aproximadamente $15 \%$ de estômagos vazios e consideraram essa freqüência relativamente baixa. Inversamente, registraram uma tendência de redução no número de estômagos vazios durante a enchente, período da 
reprodução. Já na represa de Balbina e no alto rio Solimões, o número de estômagos vazios aumentou durante o período reprodutivo de $C$. monoculus (Alcántara \& Guerra, 1988).

A alta freqüência de estômagos vazios do C. monoculus, neste estudo, parece comum a peixes piscívoros neotropicais. Lowe-McConnell (1969) examinando o conteúdo estomacal do $C$. ocellaris, na Guiana e Winemiller et al. (1997) analisando três espécies do Gênero Cichla, no sul da Venezuela, encontraram, em média, $48,3 \%$ e $71,6 \%$ de estômagos vazios, respectivamente. Goulding (1980) encontrou 62,5\% de estômagos vazios em peixes piscívoros no rio Madeira; e Leite (1987) encontrou $70 \%$ no rio Uatumã. Peixes piscívoros do reservatório de Itaipu, no rio Paraná, apresentaram $72,4 \%$ de estômagos vazios, durante um ano de amostragens (FUEM, 1985). Essa quantidade de estômagos vazios parece ser alta, mas é justificada pelo fato dos peixes piscívoros não se alimentarem continuamente, o que aumenta a probabilidade de serem capturados nesta situação. Jobling (1981) argumenta que o hábito dos peixes piscívoros de alimentarem-se uma ou duas vezes por dia é suficiente para manter a sua taxa de crescimento ótima.

A presença ou ausência de um item alimentar no estômago de um peixe predador pode estar relacionada à sua disponibilidade no ambiente (Nikolsky, 1978) e/ou a variações ontogenéticas (Wootton, 1990), embora algumas vezes, indivíduos de uma mesma população, e amostrados na mesma época e local, possam ter dietas diferentes. Na maioria dos casos o tamanho das presas tem relação direta com o tamanho de peixes predadores (Wootton, 1990).

C. monoculus não apresentou um padrão constante na dieta. Durante a cheia, ele predou peixes de hábitos pelágicos e camarão. $\mathrm{Na}$ seca, peixes bentônicos, como loricariídeos, calichitídeos e eritrinídeos, tiveram uma participação maior na dieta. Apenas tucunarés menores que $35 \mathrm{~cm}$ predaram camarões. Peixes e camarões foram selecionados por tamanho e em média mediam $29 \%$ do comprimento do tucunaré.

A abundância de camarões, no lago dos Reis, aumenta na seca (Odinetz-Collart \& Moreira, 1993). Assim a disponibilidade de camarões não se correlacionou com sua freqüência na dieta de C. monoculus. Uma explicação alternativa seria que durante a seca, os camarões ficam no fundo e são menos capturados pelo $C$. monoculus. A ausência de camarão na dieta de peixes maiores que $35 \mathrm{~cm}$ está possivelmente relacionada ao comprimento máximo atingido por essa presa, os tucunarés maiores que $35 \mathrm{~cm}$ preferem presas que medem mais de 8 $\mathrm{cm}$ e os camarões raramente ultrapassam esse comprimento (Odinetz-Collart \& Moreira, 1993). O aumento na freqüência de peixes bentônicos na dieta quando o nível da água está baixo, sugere uma dependência visual do tucunaré para predação. Nesta época a profundidade diminui (Odinetz-Collart $\&$ Moreira, 1993) e a luz penetra em toda a coluna d'água, permitindo o tucunaré explorá-la melhor. 
Em outros locais da bacia amazônica, como também em outras bacias hidrográficas, as dietas de $C$. monoculus, menores que $35 \mathrm{~cm}$, foram semelhantes, consistindo principalmente de peixes, apesar de ocorrerem algumas diferenças regionais. No alto rio Solimões os peixes foram as presas mais freqüente $(41,4 \%)$, seguido por resto de macrófitas aquáticas $(11,63 \%)$, decápodos $(6,05 \%)$ e insetos $(5,12 \%)$ (Cala et al., 1996). No rio Negro, peixes também foram a principal presa (Goulding et al., 1988). No lago da Hidrelétrica de Balbina, Oliveira (1998) observou basicamente 2 espécies de Characiformes, Hemiodopsis goeldii $\mathrm{e}$ Hemigrammus levis, em mais de $90 \%$ dos estômagos. No rio Machado, durante a cheia do rio a dieta consistiu de Serrasalmus rhombeus, Crenicicha, Semaprochilodus, Triportheus, Brycon, Schizodon e Leporinus; mas na seca somente Serrasalmus rhombeus e loricariídeos (Goulding, 1980). Nas Guianas, Lowe-McConnell (1969) encontrou uma pequena quantidade de camarão na dieta de $C$. ocellaris menores que $13 \mathrm{~cm}$, mas indivíduos maiores que $18 \mathrm{~cm}$ se alimentavam exclusivamente de peixes. Na Bacia do Orinoco, durante a estação de seca, o principal item alimentar identificado foi composto de peixes das ordens Characiformes, Perciformes e Siluriformes, com freqüência média de $78,1 \%, 14,1 \%$ e $7,8 \%$, respectivamente (Winemiller et al., 1997). Uma exceção ocorreu num reservatório natural, onde Peixoto (1982) registrou camarão como o principal alimento (84\%), seguido de peixes nos estômagos do C. ocellaris, menores que $37,5 \mathrm{~cm}$.

O tucunaré procura aumentar o comprimento de suas presas conforme seu crescimento. A relação máxima entre o comprimento do peixe-presa e comprimento do tucunaré foi de 0,37 . Esta relação parece ser variável. Winemiller et al. (1997) encontrou resultados ligeiramente mais altos em C. temensis, e Goulding (1980) registrou presas medindo até $57 \%$ do comprimento do C. ocellaris no rio Madeira.

O número mediano de presas no estômago foi igual a um, apesar de encontrarmos até oito indivíduos nos estômagos de alguns indivíduos, nestas situações as presas estavam em estágios de digestão semelhantes. Provavelmente os indivíduos não capturaram alimento continuamente, mas comeram durante períodos restritos do dia.

A análise do ritmo alimentar de C. monoculus corrobora este argumento. O tucunaré foi predominantemente diurno, e o peso do seu conteúdo estomacal apresentou dois picos diários, um pela manhã, e outro no final da tarde e início da noite, entre 16:00 e 19:00 horas $(28,1 \%)$, independente da estação hidrológica.

A quantidade de alimento consumido diariamente depende da velocidade que esse alimento é evacuado pelo estômago. A taxa de evacuação gástrica do C. monoculus, foi de $16,9 \% \mathrm{~h}^{-1}$ e o tempo de evacuação necessário para que o estômago fosse considerado vazio, isto é, com $10 \%$ do peso inicial do 
alimento, foi de 11 horas e 18 minutos.

Existem variáveis que podem influenciar na taxa de evacuação gástrica, como: o tamanho da refeição, o tamanho do predador, as características da presa e a temperatura. $\mathrm{O}$ tempo de evacuação gástrica pode decair com o tamanho do predador, quando o tamanho das presas é mantido fixo. No presente estudo a relação entre o tamanho do predador e da presa foi aproxima-damente constante, assim o possivel efeito do tamanho do predador foi anulado. Procedimento semelhante foi adotado por Swenson \& Smith (1973) e Andersen (1999).

O tipo de presa também pode afetar na taxa de evacuação gástrica (Salvanes et al., 1995), e este efeito é mais importante quando presas diferentes, como invertebrados e peixes, são considerados (Bromley, 1994). Porém, pequenas diferenças entre as presas, como por exemplo, diferentes espécies de peixes, não parecem afetar muito a taxa de evacuação gástrica. Andersen (1999) encontrou um pequeno efeito na taxa de evacuação gástrica em Meriangius merlangus, alimentado com cinco espécies de peixes, mas apenas quando a quantidade de gordura nos peixes era alta. Como os peixes predados e usados no experimento foram juvenis, e como estes apresentam uma quantidade de gordura muito baixa (Junk, 1985), é pouco provável que o uso de Tilapia rendalii para determinar a taxa de evacuação gástrica do $C$. monoculus silvestre acrescente algum vício que altere na sua estimativa.

A temperatura é um dos principais fatores abióticos que controla a taxa de metabolismo em peixes, influenciando na alimentação e nos processos digestivos (Fange \& Grove, 1979; Smith, 1989). Vários estudos mostraram a influência da temperatura sobre a taxa de evacuação gástrica (Elliot, 1972; Elliot \& Persson, 1978; Persson, 1979; Bromley, 1994; Andersen, 1998).

Neste estudo, a taxa de evacuação gástrica foi independente da variação da temperatura, possivelmente devido a pequena variação apresentada durante os experimentos $\left(2,5^{\circ} \mathrm{C}\right)$. Como neste estudo a amplitude da temperatura usada nos experimentos foi semelhante à encontrada nos lagos estudados (Odinetz-Collart \& Moreira, 1993), a taxa de evacuação gástrica estimada experimentalmente pode ser extrapolada para estes ambientes.

O material usado para estimar o peso médio do conteúdo estomacal foi fixado com formol a $10 \%$. O procedimento de fixação poderia influenciar no peso das presas, aumentando o peso médio do conteúdo estomacal e assim afetar a estimativa do consumo de alimento. Este vício, caso tenha ocorrido, foi pequeno. Billy (1992) mostrou que o peso do material fixado aumenta nos dias imediatamente posteriores a adição da formalina, mas voltam a valores próximo ao peso fresco cinco dias após serem transferidos para álcool $70 \%$. Um aumento de 5\% devido a fixação do material pode ocorrer. Este aumento, entretanto, é negligível e nenhuma correção foi usada nos dados. $\mathrm{O}$ consumo diário de alimento do 
C. monoculus estimados através dos modelos de Elliot \& Persson (1978) e de Eggers (1979) não foram diferentes nas quatro estações hidrológicas, e os valores médios foram $2,21 \%$ (erro padrão $= \pm 0,17$ ) e $2,25 \%$ (erro padrão $= \pm 0,17)$ do peso corporal, respectivamente. Estes valores são relativamente baixos se comparados àqueles relatados por Pandian \& Vivenkanandan (1985) para peixes tropicais $(4,1$ a $36 \%)$ ou mesmo registrados em outros peixes piscívoros.

Quando a taxa de consumo de alimento é comparada ao crescimento anual registrado na Amazônia Central, nota-se que não é baixa. Um C. monoculus com dois anos de idade $(2+)$ cresce aproximadamente $300 \mathrm{~g}$ ano $^{-1}$ (Correa, 1998). A taxa de conversão alimentar seria então igual a 0,11 (grama de peixe / grama de alimento) ou $11 \%$. Este valor também é inferior ao apresentado para outros piscívoros com táticas de caça semelhante, como, Perca flavescens e Stizostedium vitreum (Swenson \& Smith, 1973; Brett, 1979; Adams et al., 1982), cuja média é 20\%. Aumentandose a taxa de consumo de alimento, reduziria a taxa de conversão alimentar a valores inferiores a $10 \%$ o que seria muito baixo para um peixe piscívoro. Isto suporta a conclusão que o consumo de alimento do tucunaré é realmente baixo e que esta espécie é menos voraz do que se considerava anteriormente.

\section{Agradecimentos}

Agradecemos aos Srs. José Fernandes Alves e Antonio Adalberto Silva pela ajuda prestada na captura dos tucunarés e à direção da Estação de UH de Balbina por ter emprestado os tanques para realizar os experimentos. Este trabalho foi parte da dissertação de mestrado do HR, no curso BADPI, INPA e teve suporte financeiro do PPI 3270 INPA/MCT e CNPq.

\section{Bibliografia citada}

Adams, S.M.; Mclean, R.B.; Huffman, M.M. 1982. Structuring of a predator population through temperature-mediated effects on prey availability. Can. J. Fish. Aquat. Sci., 39:1175-1184.

Adrian, I. D. F.; Barbieri, G. 1996. Espectro alimentar e variações sazonal e espacial na composição da dieta de Parauchenipterus galeatus L. (Siluriformes, Auchenipteridae) na região do reservatório de Itaipu, PR. Revista Brasileria de Biologia, 56: 409-422.

Alcántara, B.F.; Gerra, H. 1988. Algunas consideraciones biologicas del tucunaré Cichla ocellaris Schneider. Folia Amazonica, 1:13-24.

Andersen, N.G. 1999. The effects of predator size, temperature, and prey characteristics on gastric evacuation in whiting. Journal of Fish Biology, 54:287-301.

Araujo-Lima, C. A. R. M:; Agostinho, A. A.; Fabré, N. N.; Bicudo, C. E. M.; Matsumura Tundisi, T. 1995. Trophic aspects of fish communities in Brazilian rivers and reservoirs. In Tundisi, J. G.; Bicudo, C. E. M.; Matsumura Tundisi, T. (Eds). Limnology in Brazil. ABC/SBL, Rio de Janeiro. p.105-136.

Arcifa, M:; Meschiatti, A. J. 1993. Distribution and feeding ecology of fishes in a Brazilian reservoir Lake Monte Alegre. Interciencia, 18:302-313.

Billy, A.J. 1982. The effects of formalin and isopropyl alcohol on lenght and weight measurements of Sarotherodon mossambicus Trewavas. Journal of Fish Biology, 21:107-112.

Brett, J.R. 1979. Environmental factors and growth. In Hoar, W.S.; Randall, D.J. (Eds). Fish Physiology. Academic Press, 
London. p.599-675.

Brodeur, R. D.; Pearcy, W. G. 1987. Diel feeding chronology, gastric evacuation and estimated daily ration of juvenile coho salmon, Oncorhinchus kisutch (Walbaum), in the coastal marine environment. Journal of Fish Biology, 31:465-478.

Bromley, P.J. 1994. The role of gastric evacuation experiments in quantifying the feeding rates of predatory fish. Reviews in Fish Biology and Fisheries, 4:36-66.

Cala, P.; González, E.; Varona, M. P. 1996. Aspectos biologicos y taxonomicos del tucunaré, Cichla monoculus (Pisces: Cichlidae). Dalia, 1:23-37.

Correa, R.O. 1998. Crescimento de C. monoculus (Perciformes; Cichlidae) em ambiente natural: seleção da melhor estrutura para determinação da idade. Dissertação de mestrado, INPA/UA. Manaus. 70 p.

Doble, B. D.; Eggers, D. M. 1978. Diel feeding chronology, rat of gastric evacuation, daily ration and prey selectivity in lake Washington of juvenile sockeye salmon (Onchorhynchus nerka). Transactions of American Fisheries Society, 107:36-45.

Eggers, D. M. 1979. Comments on some recent methods for estimating food consumption by fish. J. Fish. Res. Board Can., 36:1018-1019.

Elliott, J. M. 1972. Rates of gastric evacuation in brown trout, Salmo trutta L. Freshwater Biology, 2:1-18.

Elliott, J. M.; Persson, L. 1978. The Estimation of daily rates of food consumption for fish. Journal of Animal Ecology, 47:977-991.

Fange, R.; Grove, D. 1979. Digestion. In Hoar, W.J.; Randall, D.J. (Eds). Fish Physiology. Academic Press, London. p.161-260.

Fuem 1985. Relatório preliminar do projeto: Ecologia de populações de peixes no reservatório de Itaipú nos primeiros anos de sua formação-3a etapa. Fundação Universidade Estadual de Maringá, Maringá.

Gerking, S. D. 1994. Feeding ecology of fish. Academic Press, San Diego. 416 p.

Goulding, M. 1980. The Fishes and the For- est: Explorations in Amazonian natural history. University of California Press, Los Angeles. 200 p.

Goulding, M.; Carvalho, M. L.; Ferreira, E. J. G. 1988. Rio Negro. Rich life in poor water. SPB Academic Publishing, The Hague. 200 p.

Hall, S. J. 1987. Maximum daily ration and the pattern of food consumption in haddock, Melanogrammus aeglefinus L., and dab, Limanda limanda L. Journal of Fish Biology, 31:479-491.

Hobson, E. S.; Chess, J. R. 1973. Feeding oriented movement of the atherinid fish Pranesus pinguis at Majuro atoll, Marshal Islands. Fishery Bulletin, 69:491-523.

Jenkin Jr., T. M. 1969. Night feeding of brown and rainbow trout in an experimental stream channel. J. of Fish Res. Bd. Canada, 26:3275-3278.

Jobling, M. 1981. Mathematical models of gastric emptying and the estimation of daily rates of food consumption for fish. Journal of Fish Biology, 19:245-257.

Jobling, M. 1986. Mythical models of gastric emptying and implications for food consumption studies. Environmental Biology of Fishes, 16:35-50.

Jobling, M. 1987. Influences of food particle size and dietary energy content on patterns of gastric evacuation in fish test of a physiological model of gastric emptying. Journal of Fish Biology, 30:299-314.

Junk, W.J. 1985. Temporary fat storage, and adaptation of some fish species to the waterlevel fluctuations and related environmental changes of the Amazon River. Amazoniana, I X:315-351.

Leite, R.A.N. 1993. Efeitos da Usina Hidroelétrica de Tucurui sobre a composição da ictiofauna das pescarias experimentais de malhadeiras realizadas no baixo rio Tocantins (Pará.) Dissertação de mestrado, INPA/FUA. Manaus. 133 p.

Lowe-Mcconnell, R. 1969. The cichlid fishes of Guyana, South America, with notes on their ecology and breeding behaviour. Zoological Joumal of the Linnean Soci- 
ety, 48:255-302.

Lowe-McConnell, R.H. 1987. Ecological studies in tropical fish communities. Cambridge University Press, Cambridge. 382p.

Mérona, B.; Bittencourt, M.M. 1993. Fish communities of the lago do Rei, a floodplain lake in the Central Amazon. A general description. Amazoniana, XII:415-442.

Moreno-Amich, R. 1996. Feeding habits of longfin gurnard Aspitrigla obscura L., along the Catalan coast (north-western Mediterranean). Hidrobiologia, 324:219-228.

Mullen, A. J. 1986. The effect of the choice of evacuation model on the estimation of feeding rate. Environmental Biology of Fishes, 16:213-217.

Ney, J. J. 1990. Trophic economics in fisheries: assessment of demand-supply relatioships between predators and prey. Reviews in Aquatic Sciences, 2:55-81.

Nikolsky, G. V. 1978. The ecology of fishes, T.F.H. Publications, Neptune City. 352 p.

Noakes, D. L. G. 1986. Decision making in sticklebacks, Gasterosteus aculeatus. Environmental Biology of Fishes, 16:95-104.

Odinetz Collart, O.; Moreira, L. C. 1993. Potencial pesqueiro de Macrobrachium amazonicum na Amazônia Central (Ilha do Careiro): variação da abundância e do comprimento. Amazoniana, XII:399-413.

Oliveros, O. B.; Rossi, L. M. 1991. Ecologia trofica de Hoplias malabaricus malabaricus (Pisces, Erythrinidae). Revista de la Asociacion de Ciencias Naturales del Litoral, 22:55-68.

Olson, R. J.; Mullen, A. J. 1986. Recent developments for making gastric evacuation and daily ration determinations. Environmental Biology of Fishes, 16:183-191.

Pandian, T. J.; Vivekanandan, E. 1985. Energetics of feeding and digestion. In Tytler, P.; Calow, P. (Eds). Fish Energetics. Chrom Helm, London, p.99-123.

Pauly, D. 1994. Quantitative analysis of published data on the growth, metabolism, food consumption, and related features of the red-bellied piranha, Serrasalmus nattereri (Characidae). Environmental Biology of Fishes, 41:423-437.

Peixoto, J.T. 1982. Alimento de tucunaré Cichla ocellaris Bloch \& Schneider, no açude Lima Campos, Icó, Ceará. Coletanea de trabalhos técnicos, pesca e piscicultura, Minter/Dnocs, 1c:161-170.

Persson, L. 1979. The effects of temperature and diffrent food organisms on the rate of gastric evacuation in perch (Perca fluviatilis). Freshwater Biology, 9:99-104.

Persson, L. 1986. Patterns of food evacuation in fishes: a critical review. Environmental Biology of Fishes, 16:51-58.

Resende, E. K.; Pereira, R. A. C.; Almeida, V. L. L.; Silva, A. G. D. 1996. Alimentação de peixes carnívoros da planície inundável do rio Miranda, Pantanal, Mato Grosso do Sul, Brasil. EMBRAPA-CPAP. Boletim de Pesquisa, 3:5-36.

Salvanes, A. G. V.; Aksnes, D. L.; Giske, J. 1995. A surface-dependent gastric evacuation model for fish. Journal of Fish Bio$\log$, 47:679-695.

Santos, G.M. 1980. Estudo da reprodução e hábitos reprodutivos de Scizodon fasciatus, Rhytiodus microlepis $\mathrm{e}$ Rhytiodus argenteofuscus (Pisces, Anostomidae) do lago Janauacá. Acta Amazonica, 10:391-400.

Shimamoto, N.; Watanabe, J. 1994. Comparative ecology of the African pike Hepsetus odoe, and tigerfish Hydrocynus forskahlii, en the Zambezi River floodplain. Journal of Fish Biology, 45:211-225.

Singh-Renton, S.; Bromley, P. J. 1996. Effects of temperature, prey tipe and prey size on gastric evacuation in small cod and whiting. Journal of Fish Biology, 49:702-713.

Smith, R. R. 1989. Nutritional energetics. In Halver, J. E. (Ed). Fish Nutrition. Academic Press, San Diego. p.2-31.

Swenson, W. A.; Smith Jr, L. L. 1973. Gastric digestion, food consumption, feeding peridicity, and food conversion eficiency in walleye (Stizostedion vitreum vitreum). Journal of Fisheries Research Board of Canada, 30:1327-1336. 
Winemiller, K. O.; Taphorn, D. C.; BarbarinoDuque, A. 1997. Ecology of Cichla (Cichlidae) in two blackwater rivers of Southern Venezuela. Copeia, 1997:690-696.

Wootton, R. J. 1990. Ecology of teleost fishes. Chapman and Hall, London. 404 p.

Worthmann, H.; Oliveira, J. L. 1987. Comparative nutritional analysis of two sciaenidian species, the pescadas, Plagioscion squamosissimus Heckel and Plagioscion monti Soares, from different water systems of the central Amazon. Animal Research and Development, 25:7-34.

Zaret, T. M. 1980. Life history and growth relationships of Cichla ocellaris, a predatory South American cichlid. Biotropica, 12:144-157.

Aceito para publicação em 30/08/2002. 